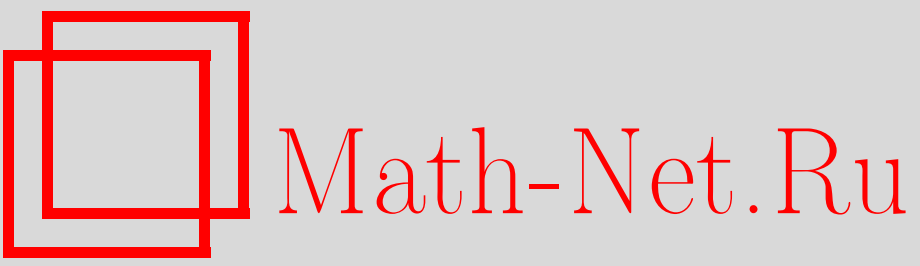

А. И. Кожанов, Т. П. Плеханова, Краевые задачи для одного класса уравнений составного типа с волновым оператором в старшей части, Итоги науки и техн. Сер. Соврем. мат. и ее прил. Темат. обз., 2020, том 188, 76-83

DOI: https://doi.org/10.36535/0233-6723-2020-188-76-83

Использование Общероссийского математического портала Math-Net.Ru подразумевает, что вы прочитали и согласны с пользовательским соглашением

http://www.mathnet.ru/rus/agreement

Параметры загрузки:

IP: 54.147 .182 .235

26 апреля 2023 г., $17: 22: 47$ 


\title{
КРАЕВЫЕ ЗАДАЧИ ДЛЯ ОДНОГО КЛАССА УРАВНЕНИЙ СОСТАВНОГО ТИПА С ВОЛНОВЫМ ОПЕРАТОРОМ В СТАРШЕЙ ЧАСТИ
}

\author{
(c) 2020 г. $\quad$ А. И. КОЖАНОВ, Т. П. ПЛЕХАНОВА
}

\begin{abstract}
АннотАция. Работа посвящена исследованию разрешимости локальных и нелокальных краевых задач для уравнений составного (соболевского) типа $D_{t}^{2 p+1}\left(D_{t}^{2}-\Delta u\right)+B u=f(x, t)$, где $D_{t}^{k}=\partial^{k} / \partial t^{k}, \Delta$ - оператор Лапласа, действующий по пространственным переменным, $B$ - дифференциальный оператор второго порядка, также действующий по пространственнным переменным, $p$ - целое неотрицательное число. Для этих уравнений доказывается существование и единственность регулярных (имеющих все обобщенные по С. Л. Соболеву производные, входящие в уравнение) решений начально-краевой задачи и краевой задачи, нелокальной по временной переменной. Описываются также некоторые обобщения и усиления полученных результатов.
\end{abstract}

Ключевые слова: уравнение составного типа, волновой оператор, начально-краевая задача, нелокальная краевая задача, регулярное решение, существование, единственность.

\section{BOUNDARY-VALUE PROBLEMS FOR ONE CLASS OF COMPOSITE EQUATIONS WITH THE WAVE OPERATOR IN THE SENIOR PART}

\author{
(c) 2020 A. I. KOZHANOV, T. P. PLEKHANOVA
}

\begin{abstract}
The work is devoted to the solvability of local and nonlocal boundary-value problems for composite (Sobolev-type) equations $D_{t}^{2 p+1}\left(D_{t}^{2}-\Delta u\right)+B u=f(x, t)$, where $D_{t}^{k}=\partial^{k} / \partial t^{k}, \Delta$ is the Laplace operator acting on spatial variables, $B$ is a second-order differential operator that also acts on spatial variables, and $p$ is a nonnegative integer. For these equations, the existence and uniqueness of regular solutions (possessing all generalized derivatives in the Sobolev sense that are involved in the equation) to initial-boundary-value problems and the boundary-value problems nonlocal in the time variable. Some generalizations and refinements of the results obtained are also described.
\end{abstract}

Keywords and phrases: composite equation, wave operator, initial-boundary-value problem, nonlocal boundary-value problem, regular solution, existence, uniqueness.

AMS Subject Classification: 35M20

1. Введение. Пусть $\Omega$ - ограниченная область пространства $\mathbb{R}_{x}^{n}$ переменных $x_{1}, \ldots, x_{n}$ с гладкой (для простоты бесконечно дифференцируемой) границей $\Gamma, Q$-цилиндр $\Omega \times(0, T)$ конечной высоты $T, S=\Gamma \times(0, T)$ - боковая граница $Q, b^{i j}(x, t), i, j=1, \ldots, n, b_{0}(x, t), f(x, t)$ - заданные функции, определенные при $(x, t) \in \bar{Q}, p$ - фиксированное целое неотрицательное число. Через $D_{t}^{k}$ будем обозначать частную производную $\partial^{k} / \partial t^{k}$ (здесь $k$ - целое неотрицательное число),

Работа выполнена при поддержке Российского фонда фундаментальных исследований (проект № 18-51-41009). 
$D_{t}^{1}=D_{t}$; через $B$ и $M_{p}$ - дифференциальные операторы, действие которых на заданной функции $v(x, t)$ определяется равенствами

$$
B v=\frac{\partial}{\partial x_{i}}\left(b^{i j}(x, t) v_{x_{j}}\right)+b_{0}(x, t) v, \quad M_{p} v=D_{t}^{2 p+1}\left(D_{t}^{2} v-\Delta v\right)+B v
$$

здесь и далее считается, что по повторяющимся индексам ведется суммирование в пределах от 1 до $n ; \Delta$ - оператор Лапласа по переменным $x_{1}, \ldots, x_{n}$.

Настоящая статья посвящена исследованию разрешимости начально-краевой задачи, а также некоторой нелокальной по временной переменной $t$ краевой задачи для дифференциальных уравнений

$$
M_{p} u=f(x, t) .
$$

Цель работы - доказательство существования и единственности регулярных решений (т.е. решений, имеющих все обобщенные по С. Л. Соболеву производные, входящие в уравнение; см. [6]) соответствующих задач.

Уравнения (1) относятся к классу уравнений составного, или соболевского, типа. Подобные уравнения активно изучаются в последнее время (см., например, $[5,10-12,14])$; интерес к этим уравнениям объясняется, с одной стороны, потребностями общей теории краевых задач для дифференциальных уравнений в частных производных, с другой-тем обстоятельством, что уравнения соболевского типа возникают во многих задачах математического моделирования (см. $[1,3,4,8,13])$.

2. Начально-краевая задача для дифференциальных уравнений составного типа с волновым оператором в старшей части. Определим вначале необходимое для изучаемой ниже начально-краевой задачи функциональное пространство. Именно, положим

$$
\begin{aligned}
V_{0}=\left\{v(x, t): D_{t}^{k} v(x, t) \in L_{\infty}\left(0, T ; W_{2}^{2}(\Omega) \cap \stackrel{\circ}{W_{2}^{1}}(\Omega)\right), k=0, \ldots, 2 p+1,\right. \\
\left.\quad D_{t}^{k} v(x, t) \in L_{\infty}\left(0, T ; L_{2}(\Omega)\right), k=2 p+2,2 p+3\right\} .
\end{aligned}
$$

Множество $V_{0}$ является линейным пространством. Зададим в этом пространстве норму

$$
\|v\|_{V_{0}}=\left(\sum_{k=0}^{2 p+1}\left\|D_{t}^{k} v\right\|_{L_{\infty}\left(0, T ; W_{2}^{2}(\Omega) \cap \stackrel{\circ}{W_{2}^{1}(\Omega)}\right.}^{2}+\left\|D_{t}^{2 p+3} v\right\|_{L_{\infty}\left(0, T ; L_{2}(\Omega)\right)}^{2}\right)^{1 / 2} .
$$

Очевидно, что множество $V_{0}$ с данной нормой представляет собой банахово пространство.

Начально-краевая задача: найти функиию $u(x, t)$, являющуюся решением уравнения (1) в иилиндре $Q$ и удовлетворяюшуюо условиям

$$
\begin{gathered}
\left.u(x, t)\right|_{S}=0, \\
\left.D_{t}^{k} u(x, t)\right|_{t=0}=0, \quad k=0, \ldots, 2 p+2, \quad x \in \Omega .
\end{gathered}
$$

Теорема 1. Пусть выполняются условия

$$
b^{i j}(x, t) \in C^{1}(\bar{Q}), \quad i, j=1, \ldots, n, \quad b_{0}(x, t) \in C(\bar{Q}),
$$

а также одно из условий

$$
\begin{gathered}
f(x, t) \in L_{2}(Q), \quad f_{t}(x, t) \in L_{2}(Q) ; \\
f(x, t) \in L_{2}\left(0, T ; \stackrel{\circ}{W}_{2}^{1}(\Omega)\right) .
\end{gathered}
$$

Тогда начально-краевая задача (1)-(3) имеет в пространстве $V_{0}$ единственное решение.

Доказательство. Воспользуемся методом регуляризации и методом продолжения по параметру.

Пусть $\varepsilon-$ положительное число. Рассмотрим следующую краевую задачу: найти функиию $u(x, t)$, являюшуюся решением уравнения

$$
M_{p} u-\varepsilon D_{t}^{2 p+2} \Delta u=f(x, t)
$$


в иилиндре $Q$ и удовлетворяюшуюо условиям (2) и (3).

Определим пространство $V_{1}$ и норму в нем:

$$
\begin{aligned}
& V_{1}=\left\{v(x, t): D_{t}^{k} v(x, t) \in L_{\infty}\left(0, T ; W_{2}^{2}(\Omega) \cap \stackrel{\circ}{W_{2}^{1}}(\Omega)\right), k=0, \ldots, 2 p+1,\right. \\
& \left.D_{t}^{2 p+2} v(x, t) \in L_{2}\left(0, T ; W_{2}^{2}(\Omega) \cap \stackrel{\circ}{W}_{2}^{1}(\Omega)\right), D_{t}^{2 p+3} v(x, t) \in L_{2}\left(0, T ; L_{2}(\Omega)\right)\right\}, \\
& \|v\|_{V_{1}}=\left(\sum_{k=0}^{2 p+1}\left\|D_{t}^{k} v\right\|_{L_{\infty}\left(0, T ; W_{2}^{2}(\Omega) \cap \stackrel{\circ}{W_{2}^{1}(\Omega)}\right)}^{2}\right. \\
& \left.\quad+\left\|D_{t}^{2 p+2} v\right\|_{L_{2}\left(0, T ; W_{2}^{2}(\Omega) \cap \stackrel{\circ}{1}_{2}^{1}(\Omega)\right)}^{2}+\left\|D_{t}^{2 p+3} v\right\|_{L_{2}\left(0, T ; L_{2}(\Omega)\right.}^{2}\right)^{1 / 2} .
\end{aligned}
$$

Очевидно, что множество $V_{1}$ с этой нормой является банаховым пространством.

Покажем, что при фиксированном $\varepsilon$ и при $f(x, t) \in L_{2}(Q)$ краевая задача $\left(1_{\varepsilon}\right),(2),(3)$ разрешима в пространстве $V_{1}$.

Пусть $\lambda \in[0,1]$. Рассмотрим следующую краевую задачу: найти функиию $u(x, t)$, являюшуюся решением уравнения

$$
D_{t}^{2 p+3} u-\varepsilon D_{t}^{2 p+2} \Delta u-\lambda\left[D_{t}^{2 p+1} \Delta u-B u\right]=f(x, t)
$$

в иилиндре $Q$ и удовлетворяюшую условиям (2) и (3).

Заметим прежде всего, что краевая задача $\left(1_{\varepsilon, 0}\right),(2),(3)$ разрешима в пространстве $V_{1}$. Далее, покажем, что для всевозможных решений $u(x, t)$ краевой задачи $\left(1_{\varepsilon, \lambda}\right),(2),(3)$ из пространства $V_{1}$ имеет место априорная оценка

$$
\|u\|_{V_{1}} \leqslant R_{0}\|f\|_{L_{2}(Q)}
$$

с постоянной $R_{0}$, определяемой лишь функциями $b^{i j}(x, t), i, j=1, \ldots, n, b_{0}(x, t)$, областью $\Omega$ и числами $T$ и $\varepsilon$.

Рассмотрим равенство

$$
\int_{0}^{t} \int_{\Omega}\left\{D_{\tau}^{2 p+3} u-\varepsilon D_{\tau}^{2 p+2} \Delta u-\lambda\left[D_{\tau}^{2 p+1} \Delta u-B u\right]\right\} D_{\tau}^{2 p+2} u d x d \tau=\int_{0}^{t} \int_{\Omega} D_{\tau}^{2 p+2} u f d x d \tau .
$$

Интегрируя по частям с использованием условий (2) и (3), используя неравенства

$$
\int_{\Omega}\left[D_{t}^{k} u(x, t)\right]^{2} d x \leqslant T \int_{0}^{t} \int_{\Omega}\left[D_{\tau}^{k+1} u(x, \tau)\right]^{2} d x d \tau, \quad k=0, \ldots, 2 p+1,
$$

и применяя лемму Гронуолла, получим, что для решений $u(x, t)$ краевой задачи $\left(1_{\varepsilon, \lambda}\right),(2),(3)$ выполняется оценка

$$
\int_{\Omega}\left[D_{t}^{2 p+2} u(x, t)\right]^{2} d x+\sum_{i=1}^{n} \int_{0}^{t} \int_{\Omega}\left[D_{\tau}^{2 p+2} u_{x_{i}}\right]^{2} d x d \tau \leqslant R_{1}\|f\|_{L_{2}(Q)}^{2}
$$

с постоянной $R_{1}$, определяемой лишь функциями $b^{i j}(x, t), i, j=1, \ldots, n, b_{0}(x, t)$, областью $\Omega$ и числами $T$ и $\varepsilon$.

Анализируя аналогичным образом равенство

$$
-\int_{0}^{t} \int_{\Omega}\left\{D_{\tau}^{2 p+3} u-\varepsilon D_{\tau}^{2 p+2} \Delta u-\lambda\left[D_{\tau}^{2 p+1} \Delta u-B u\right]\right\} D_{\tau}^{2 p+2} \Delta u d x d \tau=-\int_{0}^{t} \int_{\Omega} f D_{\tau}^{2 p+2} \Delta u d x d \tau
$$


т.е. вновь используя граничные условия (2) и (3), неравенства (9) и применяя лемму Гронуолла, получим, что для решений $u(x, t)$ краевой задачи eqrefeq1el, (2), (3) выполняется вторая априорная оценка

$$
\sum_{i=1}^{n} \int_{\Omega}\left[D_{t}^{2 p+2} u_{x_{i}}(x, t)\right]^{2} d x+\int_{0}^{t} \int_{\Omega}\left[D_{\tau}^{2 p+2} \Delta u\right]^{2} d x d \tau \leqslant R_{2}\|f\|_{L_{2}(Q)}^{2},
$$

постоянная $R_{2}$ в которой вновь определяется функциями $b^{i j}(x, t), i, j=1, \ldots, n, b_{0}(x, t)$, областью $\Omega$ и числами $T$ и $\varepsilon$.

Из оценок (10) и (12) и вытекает требуемая оценка (7).

Согласно теореме о методе продолжения по параметру (см. [гл. II, § 14]] [7]) разрешимость в пространстве $V_{1}$ краевой задачи $\left(1_{\varepsilon, 0}\right),(2),(3)$, а также наличие априорной оценки $(7)$ и дадут при фиксированном $\varepsilon$ разрешимость в том же пространстве краевой задачи $\left(1_{\varepsilon, \lambda}\right),(2),(3)$ при всех $\lambda$ из отрезка $[0,1]$. Это и означает, что краевая задача $\left(1_{\varepsilon}\right),(2),(3)$ разрешима в требуемом пространстве.

Покажем теперь, что для решений $u(x, t)$ краевой задачи $\left(1_{\varepsilon}\right),(2),(3)$ имеют место равномерные по $\varepsilon$ априорные оценки.

Вновь рассмотрим равенство (8), но теперь при $\lambda=1$. Интегрируя по частям, используя краевые условия, неравенства (9) и применяя лемму Гронуолла, получим, что для решений $u(x, t)$ краевой задачи $\left(1_{\varepsilon}\right),(2),(3)$ из пространства $V_{1}$ будет иметь место оценка

$$
\int_{\Omega}\left[D_{t}^{2 p+2} u(x, t)\right]^{2} d x+\sum_{i=1}^{n} \int_{\Omega}\left[D_{t}^{2 p+1} u_{x_{i}}(x, t)\right]^{2} d x+\varepsilon \sum_{i=1}^{n} \int_{0}^{t} \int_{\Omega}\left[D_{\tau}^{2 p+2} u_{x_{i}}\right]^{2} d x d \tau \leqslant R_{3}\|f\|_{L_{2}(Q)}^{2}
$$

с постоянной $R_{3}$, определяемой лишь функциями $b^{i j}(x, t), i, j=1, \ldots, n, b_{0}(x, t)$, областью $\Omega$ и числом $T$.

На следующем шаге рассмотрим равенство (11) при $\lambda=1$. Преобразуя левую часть этого равенства с помощью интегрирования по частям, а правую часть - интегрированием по частям по переменной $t$ при выполнении условия (5) или по пространственным переменным при выполнении условия (6), нетрудно после использования неравенств (10), второго основного неравенства для эллиптических операторов (см. [2, гл. III, § 8]), а также леммы Гронуолла получить оценку

$$
\sum_{i=1}^{n} \int_{\Omega}\left[D_{t}^{2 p+2} u_{x_{i}}(x, t)\right]^{2} d x+\int_{\Omega}\left[D_{t}^{2 p+1} \Delta u(x, t)\right]^{2} d x+\varepsilon \int_{0}^{t} \int_{\Omega}\left[D_{\tau}^{2 p+2} \Delta u\right]^{2} d x d \tau \leqslant R_{4}
$$

в которой число $R_{4}$ определяется функциями $b^{i j}(x, t), i, j=1, \ldots, n, b_{0}(x, t)$, областью $\Omega$, числом $T$, а также нормами функций $f(x, t)$ и $f_{t}(x, t)$ в $L_{2}(Q)$ при выполнении условия (5), или же нормами функций $f(x, t)$ и $f_{x_{i}}(x, t), i=1, \ldots, n$, в $L_{2}(Q)$ при выполнении условия $(6)$.

Последняя оценка

$$
\int_{0}^{t} \int_{\Omega}\left[D_{\tau}^{2 p+3} u\right]^{2} d x d \tau \leqslant R_{5}
$$

с постоянной $R_{5}$, не зависящей от $\varepsilon$, очевидным образом вытекает из оценки (14).

Полученных оценок (13)-(15) уже вполне достаточно для организации предельного перехода. Выбирая такую числовую последовательность $\left\{\varepsilon_{m}\right\}_{m=1}^{\infty}$, что $\varepsilon_{m} \rightarrow 0$ при $m \rightarrow \infty$, и далее выбирая из последовательности $\left\{u^{\varepsilon_{m}}(x, t)\right\}_{m=1}^{\infty}$ решений краевой задачи $\left(1_{\varepsilon_{m}}\right),(2),(3)$ слабо сходящуюся подпоследовательность (с помощью свойства рефлексивности гильбертова пространства), нетрудно получить, что предельная функция $u(x, t)$ принадлежит пространству $V_{0}$ и представляет собой искомое решение начально-краевой задачи (1)-(3). 
Единственность в пространстве $V_{0}$ решений начально-краевой задачи (1)-(3) очевидным образом вытекает из анализа равенства

$$
\int_{0}^{t} \int_{\Omega} M_{p} u D_{\tau}^{2 p+2} u d x d \tau=0 .
$$

Теорема доказана.

3. Нелокальная краевая задача для дифференциальных уравнений составного типа с волновым оператором в старшей части. В настоящем разделе будет изучена разрешимость нелокальной по временной переменной задачи для некоторых модельных уравнений (1) в случае $p=1$. О более общих уравнениях будет сказано в следующем разделе.

Пусть $\alpha_{k}, k=0,1,2$, - заданные действительные числа.

Нелокальная краевая задача: найти функиию $u(x, t)$, являюшуюся решением уравнения $M_{1} u=f(x, t)$ в иилиндре $Q$ и удовлетворяющую условию (2), а такэсе условиям

$$
\left.D_{t}^{k} u(x, t)\right|_{t=0}=\left.\alpha_{k} D_{t}^{k} u(x, t)\right|_{t=T}, \quad k=0,1,2, \quad x \in \Omega .
$$

В случае $\alpha_{k}=0$ задача (1), (2), (16) будет совпадать с начально-краевой задачей (1)-(3), вместе с тем даже в модельном случае при $\alpha_{k} \neq 0$ условия ее разрешимости будут отличаться от условий теоремы 1.

Пусть $v(x)$ есть функция из пространства $\stackrel{\circ}{W} \frac{1}{2}(\Omega)$. Имеет место неравенство

$$
\int_{\Omega} v^{2}(x) d x \leqslant d_{0} \sum_{i=1}^{n} \int_{\Omega} v_{x_{i}}^{2}(x) d x,
$$

постоянная $d_{0}$ в которых определяется лишь областью $\Omega$ (считается, что число $d_{0}$ здесь выбрано минимальным среди возможных) - см. [ [7], гл. II].

Далее, пусть $w(t)$ есть функция из пространства $W_{2}^{1}([0, T])$ такая, что для нее выполняется условие $w(0)=\alpha_{0} w(T)$. Тогда выполняется неравенство

$$
w^{2}(T) \leqslant \frac{T}{\left(1-\alpha_{0}\right)^{2}} \int_{0}^{T} w^{\prime 2}(t) d t .
$$

Неравенства нам понадобятся ниже.

Пусть $\gamma_{1}, \gamma_{2}, \bar{b}_{0}$ и $b_{1}$ есть фиксированные действительные числа (роль этих чисел будет определена ниже) и пусть $F_{1}\left(\gamma_{1}, \gamma_{2}, b_{1}, \xi\right)$ и $F_{2}\left(x, \gamma_{1}, \gamma_{2}, \bar{b}_{0}, b_{1}, \xi\right)$ есть квадратичные по $\xi$ при $\xi \in \mathbb{R}^{2}$ формы

$$
\begin{gathered}
F_{1}\left(\gamma_{1}, \gamma_{2}, b_{1}, \xi\right)=\frac{\gamma_{1}\left(1-\alpha_{1}^{2}\right)}{2} \xi_{1}^{2}+\left(\alpha_{0} \alpha_{1}-1\right) b_{1} \xi_{1} \xi_{2}+\frac{\gamma_{2} b_{1}\left(1-\alpha_{0}\right)^{2}}{T} \xi_{2}^{2}, \\
F_{2}\left(x, \gamma_{1}, \gamma_{2}, \bar{b}_{0}, b_{1}, \xi\right)=\frac{\left(1-\gamma_{1}\right)\left(1-\alpha_{1}^{2}\right)}{2 d_{0}} \xi_{1}^{2}+ \\
+\left[b_{0}(x, T)-\alpha_{0} \alpha_{1} b_{0}(x, 0)\right] \xi_{1} \xi_{2}+\left[\frac{\bar{b}_{0}\left(1-\alpha_{0}\right)^{2}}{T}+\frac{\left(1-\gamma_{2}\right) b_{1}\left(1-\alpha_{0}\right)^{2}}{d_{0} T}\right] \xi_{2}^{2} .
\end{gathered}
$$

Теорема 2. Пусть выполняются условия

$$
\begin{gathered}
B=b_{1} \Delta+b_{0}(x, t) \\
b_{1}>0, \quad b_{0}(x, t) \in C^{2}(\bar{Q}), \quad b_{0}(x, t) \leqslant-\bar{b}_{0}<0, \quad b_{0 t t}(x, t) \geqslant 0 \text { npu }(x, t) \in \bar{Q}, \\
\alpha_{0}^{2} b_{0 t}(x, 0)-b_{0 t}(x, T) \text { npu } x \in \bar{\Omega} \\
\alpha_{0} \neq 1, \quad\left|\alpha_{1}\right|<1, \quad\left|\alpha_{2}\right|<1
\end{gathered}
$$


существуют числа $\gamma_{1}$ и $\gamma_{2}$ такие, что $\gamma_{1} \in(0,1), \gamma_{2} \in(0,1]$, квадратичная форма $F_{1}\left(\gamma_{1}, \gamma_{2}, b_{1}, \xi\right)$ положительно определена, для квадратичной формы $F_{2}\left(x, \gamma_{1}, \gamma_{2}, \bar{b}_{0}, b_{1}, \xi\right)$ выполняется при $x \in \bar{\Omega}$ неравенство

$$
F_{2}\left(x, \gamma_{1}, \gamma_{2}, \bar{b}_{0}, b_{1}, \xi\right) \geqslant 0 .
$$

Тогда для любой функиии $f(x, t)$, для которой выполняется одно из условий (5) или (6), нелокальная задача (1), (2), (16) имеет решение $u(x, t)$, принадлежащее пространству $V_{0}$, и причем ровно одно.

Доказательство. Вновь, как и при доказательстве теоремы 1, воспользуемся методом регуляризации и методом продолжения по параметру. Не повторяя все рассуждения (о том, как вводится регуляризующий оператор, как используется метод продолжения по параметру) покажем лишь, что при выполнении условий теоремы для всевозможных решений нелокальной задачи (1), (2), (16) имеет место априорная оценка в пространстве $V_{0}$.

Рассмотрим равенство

$$
\int_{Q} M_{1} u u_{t t} d x d t=\int_{Q} f u_{t t} d x d t
$$

После интегрирования по частям это равенство преобразуется к виду

$$
\begin{aligned}
& \frac{1-\alpha_{2}^{2}}{2} \int_{\Omega} u_{t t}^{2}(x, T) d x+\frac{1-\alpha_{1}^{2}}{2} \sum_{i=1}^{n} \int_{\Omega} u_{x_{i} t}^{2}(x, T) d x+b_{1} \sum_{i=1}^{n} \int_{Q} u_{x_{i} t}^{2} d x d t+ \\
& +b_{1}\left(\alpha_{0} \alpha_{1}-1\right) \sum_{i=1}^{n} \int_{\Omega} u_{x_{i}}(x, T) u_{x_{i} t}(x, T) d x-\int_{Q} b u_{t}^{2} d x d t+ \\
& +\frac{1}{2} \int_{Q} b_{0 t t} u^{2} d x d t+\int_{\Omega}\left[b_{0}(x, T)-\alpha_{0} \alpha_{1} b_{0}(x, 0)\right] u(x, T) u_{t}(x, T) d x+ \\
& +\int_{\Omega}\left[\alpha_{0}^{2} b_{0 t}(x, 0)-b_{0 t}(x, T)\right] u^{2}(x, T) d x=\int_{Q} f u_{t t} d x d t .
\end{aligned}
$$

Используя условие (20), а также неравенства (17) и (18), нетрудно от этого равенства перейти к неравенству

$$
\begin{gathered}
\frac{1-\alpha_{2}^{2}}{2} \int_{\Omega} u_{t t}^{2}(x, T) d x+\frac{\gamma_{1}\left(1-\alpha_{1}^{2}\right)}{2} \sum_{i=1}^{n} \int_{\Omega} u_{x_{i} t}^{2}(x, T) d x+b_{1}\left(\alpha_{0} \alpha_{1}-1\right) \sum_{i=1}^{n} \int_{\Omega} u_{x_{i}}(x, T) u_{x_{i} t}(x, T) d x+ \\
+\frac{\gamma_{2} b_{1}\left(1-\alpha_{0}^{2}\right)}{T} \sum_{i=1}^{n} \int_{\Omega} u_{x_{i}}^{2}(x, T) d x+\frac{\left(1-\gamma_{1}\right)\left(1-\alpha_{1}^{2}\right)}{2 d_{0}} \int_{\Omega} u_{t}^{2}(x, T) d x+ \\
+\int_{\Omega}\left[b_{0}(x, T)-\alpha_{0} \alpha_{1} b_{0}(x, 0)\right] u(x, T) u_{t}(x, T) d x+\left[\frac{\bar{b}_{0}\left(1-\alpha_{0}\right)^{2}}{T}+\frac{\gamma_{2} b_{1}\left(1-\alpha_{0}\right)^{2}}{d_{0} T}\right] \int_{\Omega} u^{2}(x, T) d x \leqslant \\
\leqslant\left|\int_{Q} f u_{t t} d x d t\right|
\end{gathered}
$$

Условия (21) и (22) означают, что следствием (23) является неравенство

$$
\int_{\Omega} u_{t t}^{2}(x, T) d x+\sum_{i=1}^{n} \int_{\Omega} u_{x_{i} t}^{2}(x, T) d x \leqslant K_{1}\left|\int_{Q} f u_{t t} d x d t\right|,
$$

в котором число $K_{1}$ положительно и определяется лишь числами $\alpha_{0}, \alpha_{1}, \alpha_{2}, b_{1}, \bar{b}_{0}, T$, областью $\Omega$, а также функцией $b_{0}(x, t)$. 
Рассмотрим равенство

$$
\int_{Q}(T-t) M_{1} u u_{t t} d x d t=\int_{Q}(T-t) f u_{t t} d x d t
$$

Повторяя операцию интегрирования по частям, используя далее для оценки правых частей (24) и (25) неравенство Юнга и учитывая, что следствием равенства (23) и неравенства (25) будет оценка

$$
\sum_{i=1}^{n} \int_{Q} u_{x_{i} t}^{2} d x d t \leqslant K_{2}\left|\int_{Q} f u_{t t} d x d t\right|
$$

в которой число $K_{1}$ положительно и определяется лишь числами $\alpha_{0}, \alpha_{1}, \alpha_{2}, b_{1}, \bar{b}_{0}, T$, областью $\Omega$, а также функцией $b_{0}(x, t)$, получим что для решений $u(x, t)$ нелокальной задачи $(1),(2),(16)$ будет выполняться оценка

$$
\int_{Q}\left[u_{t t}^{2}+\sum_{i=1}^{n} u_{x_{i} t}^{2}\right] d x d t+\int_{\Omega}\left[u_{t t}^{2}(x, T)+\sum_{i=1}^{n} u_{x_{i} t}^{2}(x, T)\right] d x \leqslant K_{3}
$$

с постоянной $K_{3}$, определяющейся лишь числами $\alpha_{0}, \alpha_{1}, \alpha_{2}, b_{1}, \bar{b}_{0}, T$, областью $\Omega$ и функциями $b_{0}(x, t)$ и $f(x, t)$.

Повторяя теперь все выполненные выше действия для функции $\bar{u}(x, t)=\Delta u_{t t}(x, t)$, но при этом в интегралах с функцией $f(x, t)$ дополнительно интегрируя по частям (с использованием условий (5) или (6)) получим, что для решений краевой задачи (1), (2), (16) будет иметь место оценка

$$
\sum_{i=1}^{n} \int_{Q} u_{x_{i} t t}^{2} d x d t+\int_{Q}\left(\Delta u_{t}\right)^{2} d x d t+\sum_{i=1}^{n} \int_{\Omega} u_{x_{i} t t}^{2}(x, T) d x+\int_{\Omega}\left[\Delta u_{t}(x, T)\right]^{2} d x \leqslant K_{4},
$$

постоянная $K_{4}$ в которой вновь определяется лишь числами $\alpha_{0}, \alpha_{1}, \alpha_{2}, b_{1}, \bar{b}_{0}, T$, областью $\Omega$ и функциями $b_{0}(x, t)$ и $f(x, t)$.

Из оценок $(28)$ и $(29)$ и вытекает равномерная ограниченность в пространстве $V_{0}$ всевозможных решений нелокальной краевой задачи $(1),(2),(16)$. Как уже говорилось выше, из этой оценки и следует разрешимость искомой задачи в пространстве $V_{0}$.

Единственность решений очевидна.

Теорема доказана.

4. Заключение. Методы исследования разрешимости начально-краевой задачи (1)-(3) и нелокальной задачи (1), (2), (16) вполне могут быть перенесены и на более общие уравнения. Например, в п. 1 оператор Лапласа и оператор $B$ можно заменить общими эллиптическими операторами второго порядка; в п. 2 разрешимость нелокальной по временной переменной задачи нетрудно изучить и для уравнений (1) порядка $2 p+3$ (но, заметим, все выкладки и условия будут существенно более громоздкими).

\section{СПИСОК ЛИТЕРАТУРЫ}

1. Корпусов М. О. Разрушение в неклассических нелокальных уравнениях. - М.: Либроком, 2011.

2. Ладыженская О. А., Уральцева Н. Н. Линейные и квазилинейные уравнения эллиптического типа. М.: Наука, 1973.

3. Ларъкин Н. А., Новиков В. А., Яненко Н. Н. Нелинейные уравнения переменного типа. - Новосибирск: Наука, 1983.

4. Маслов В. П., Мосолов П. П. Уравнения одномерного баротропного газа. - М.: Наука, 1990.

5. Свешников А. Г., Алъшин А. Б., Корпусов М. О., Плетнер Ю. Д. Линейные и нелинейные уравнения соболевского типа. - М.: Физматлит, 2007, zbl1179.35007.

6. Соболев C. Л. Некоторые применения функционального анализа в математической физике. - М.: Наука, 1988. 
7. Треногин В. А. Функциональный анализ. - М.: Наука, 1980.

8. Умаров $X . Г$. Полугруппы операторов и точные решения задач анизотропной фильтрации. - М.: Физматлит, 2009.

9. Худавердиев К., Велиев А. Исследование одномерной смешанной задачи для одного класса псевдогиперболических уравнений третьего порядка с нелинейной операторной правой частью. - Баку: Gasioglu, 2010.

10. Demidenko G. V., Uspenskii S. V. Partial Differential Equations and Systems not Solvable with Respect to Highest Order Derivatives. - New-York: Marsel Dekker Inc., 2003, MR1831690.

11. Hayashi N, Kaikina E. I., Naumkin P. I., Shismarev I. A. Asymptotics for Dissipative Nonlinear Equation. - Berlin: Springer-Verlaq, 2006.

12. Kozhanov A. I. Composite Type Equations and Inverse Problems. - Utrecht: VSP, 1999.

13. Liu S, Triggiani R. An Inverse Problem for a Third Order PDE Arising in High-Insensity Ultrasound Global Uniqueness and Stability by one Boundary Measurement// J. of Inverse and Ill-Posed Problems - 2014. - 21, № 6. - P. 825-870.

14. Pyatkov S. G. Operator Theory Nonclassical Problems. - Utrecht: VSP, 2003.

Кожанов Александр Иванович

Институт математики им. С. Л. Соболева СО РАН, Новосибирск

E-mail: kozhanov@math.nsc.ru

Плеханова Татьяна Петровна

Бурятский государственный университет, Улан-Удэ

E-mail: tatuana575@mail.ru 